Ana Isabel Dias da Silva

Jorge Mesquita Huet Machado²

Elizabeth Gloria Oliveira Barbosa Santos ${ }^{3}$

Maria Helena Palucci Marziale ${ }^{4}$

\section{Acidentes com material biológico relacionados ao trabalho: análise de uma abordagem institucional*}

\author{
Work-related accidents with biological material: analyses on \\ an institutional approach
}

1 Tecnologista do Núcleo de Análise de Situação de Saúde (NASS), Diretoria de Recursos Humanos (DIREH) da Fundação Oswaldo Cruz (Fiocruz), Rio de Janeiro, RJ, Brasil.

2 Pesquisador da Fundação Oswaldo Cruz (Fiocruz), Diretoria Regional de Brasília, DF, Brasil.

3 Pesquisadora e Professora do Departamento de Ciências Biológicas da Escola Nacional de Saúde Pública (ENSP) da Fundação Oswaldo Cruz (Fiocruz), Rio de Janeiro, RJ, Brasil.

${ }^{4}$ Pesquisadora e Professora Titular da Escola de Enfermagem de Ribeirão Preto da Universidade de São Paulo (USP), Ribeirão Preto, SP, Brasil.

\footnotetext{
* Trabalho desenvolvido a partir da dissertação de mestrado de Ana Isabel Dias da Silva intitulada Análise da Qualidade dos Registros de Acidentes Biológicos com Materiais Perfurocortantes da Fundação Oswaldo Cruz, no período de 1999 a 2004, defendida em 2006 no Programa de Pós-Graduação em Saúde Pública da Escola Nacional de Saúde Pública (Fiocruz).

O presente trabalho não foi financiado e não apresenta conflito de interesses.

Contato:

Ana Isabel Dias da Silva

Núcleo de Análise de Situação de Saúde (NASS/Fiocruz)

Avenida Brasil, 4365, Rio de Janeiro, RJ, Brasil

CEP: $21040-360$

E-mail:

abela.ana@gmail.com
}

Recebido: 13/07/2011

Revisado: 14/10/2011

Aprovado: 21/10/2011

\section{Resumo}

Objetivo: analisar o perfil de relatórios, instrumentos de registros e fluxos de tratamento, notificações, acompanhamentos, profilaxias e encaminhamentos devidos a acidentes causados por material perfurocortante em trabalhadores de saúde em uma instituição de saúde pública. Métodos: a coleta de dados usou um questionário semiestruturado aplicado aos profissionais responsáveis pelos registros de acidentes e avaliou os formulários utilizados em três setores da instituição de estudo. Resultados: foram analisados 134 registros de acidentes biológicos. Quando comparamos os registros da instituição com os 50 registrados no mesmo período pelo banco de dados da Secretaria Municipal de Saúde do Rio de Janeiro, apenas 11 casos foram concordantes. Também identificamos falta de padronização nos formulários utilizados, fluxos diferentes e falta de registros de investigações dos casos, além de ausência de acompanhamento do desfecho dos acidentes. Conclusão: constataram-se subnotificação e discordância entre as fontes. Um formulário de registro para acidentes biológicos foi elaborado para informações de notificação e investigações, bem como criado um programa para monitorar acidentes causados por material perfurocortante, visando melhorar a vigilância de saúde dos trabalhadores da instituição.

Palavras-chave: acidentes de trabalho; riscos ocupacionais; saúde pública; vigilância; acidente perfurocortantes.

\begin{abstract}
Objective: To analyze profile of reports, recording systems, and flowcharts for treatment, notification, follow-up, prophylaxis as well as referrals concerning accidental puncture or laceration among workers from a public health institute. Method: Data was collected by semi-structured questionnaires answered by professionals in charge of recording accidents, and from reporting forms used in three departments of the institute under study. Results: One hundred and thirty-four biological accident records were analyzed. When we compared the records of the institute with other fifty records from the same period by Rio de Janeiro Municipal Health Secretariat data base, only 11 cases matched. We also observed the reporting forms were not standardized with flows differing from each other; there were no records on the investigations of accident causes or follow up of accident outcomes. Conclusion: Underreporting and discordance among the forms were observed. We elaborated a form for recording biological accidents as well as a program to monitor accidental puncture or laceration injuries, both aimed at improving worker's health surveillance of the institute.
\end{abstract}

Keywords: occupational risks; public health; work accident; surveillance; accidental puncture or laceration. 


\section{Introdução}

A exposição a acidentes biológicos com objetos perfurocortantes representa um risco à saúde dos trabalhadores, principalmente, devido à possibilidade de transmitir patógenos como o vírus da hepatite $\mathrm{B}$ (HBV), vírus da hepatite $\mathrm{C}$ (HCV) e vírus da imunodeficiência humana (HIV). As consequências dessa exposição podem afetar os trabalhadores física e emocionalmente, além de ter repercussões negativas em suas relações familiares e sociais (MARZIALE; RODRIGUES, 2002; MARZIALE, 2003; GODFRE, 2001).

Nos Estados Unidos, estima-se que mais de oito milhões de trabalhadores da saúde possam estar expostos a acidentes com materiais perfurocortantes (CENTERS FOR DISEASE CONTROL AND PREVENTION, 2004). O risco de infecção de uma agulha contaminada é de um entre três para hepatite $\mathrm{B}$, um entre 30 para hepatite $\mathrm{C}$ e um entre 300 para HIV (GODFRE, 2001). Dos 16.922 acidentes documentados de 1995 a 2001, 44\% envolveram enfermeiras, $28 \%$ médicos, $15 \%$ técnicos de laboratório, $4 \%$ estudantes e $3 \%$ pessoal de limpeza (CENTERS FOR DISEASE CONTROL AND PREVENTION, 1987). No Brasil, este número pode chegar a três milhões. Segundo recente estudo desenvolvido em um centro de referência de saúde, 39,5\% dos auxiliares de enfermagem haviam se acidentado com material biológico, corroborando a vulnerabilidade desse segmento profissional (SPAGNUOLO; BALDO; GUERRINI, 2008).

No Brasil, os acidentes de trabalho são comunicados pelos sistemas de informação da Previdência Social e do Ministério da Saúde por meio da Comunicação de Acidente de Trabalho - CAT (BRASIL, 1997), pelo Sistema de Informação de Agravos de Notificação - Sinan (BRASIL, 2007) e pelo Sistema de Informações Sobre Mortalidade SIM (BRASIL, 2000a). Os dados são inseridos nesses sistemas pelos funcionários das unidades de assistência médica.

Um trabalhador que pertença ao regime da Consolidação das Leis Trabalhistas (CLT) e sofra um acidente deve buscar ajuda médica imediatamente para receber a assistência apropriada ao evento. Neste caso, o acidente deve ser registrado e, de posse do referido registro, o médico ou os representantes legais encaminham o trabalhador para a Previdência. Quando o trabalhador pertencer ao Regime Jurídico Único (RJU), o registro é feito em um formulário da instituição à qual ele pertence. O registro com o formulário deve ser encaminhado ao departamento de recursos humanos que, por sua vez, encaminhará ao Instituto Nacio- nal de Previdência Social (INSS), conforme direcionado pela Lei no ${ }^{\circ}$ 8213/917 (BRASIL, 1998).

O campo de estudo apresentado neste artigo é uma instituição pública federal vinculada ao Ministério da Saúde, com sede na cidade do Rio de Janeiro, e que mantém presença permanente em todo o território nacional por meio de unidades descentralizadas. A instituição participa do Sistema Único de Saúde (SUS), orientada para o desenvolvimento de estratégias de saúde pública referentes ao ensino, à pesquisa e à produção de insumos (MINAYO, 1998).

Medidas de biossegurança no seu âmbito se iniciaram em 1983 através de um curso internacional, coordenado pela Organização Mundial da Saúde (OMS), que estabeleceu uma referência para o desenvolvimento e a divulgação de normas internacionais. Dois anos depois, a instituição promove o primeiro curso de biossegurança realizado para profissionais de saúde. Daí em diante, através de seu Instituto Nacional para Controle de Qualidade em Saúde (INCQS), a Fiocruz lançou um programa voltado para implementar medidas de biossegurança. Tal processo resultou na organização da Comissão Técnica de Biossegurança - CTBio (MINAYO, 1997), para identificar e prevenir os riscos em atividades de trabalho. Finalmente, com orientação da Comissão Interna de Biossegurança, foram organizados os Comitês de Biossegurança (COELHO, 2006) na perspectiva de implantação das medidas de segurança em setores da referida instituição.

As medidas de biossegurança implementadas envolvem todas as unidades da instituição que apresentam risco à saúde nas atividades de laboratório, assistência médica e outros serviços.

Em 1998, a instituição buscou planejar e racionalizar tais atividades para melhorar os procedimentos, articulando as ações de biossegurança com a gestão ambiental e a saúde do trabalhador a partir da organização de um programa institucional: “Fiocruz Saudável” (MINAYO, 1998).

Os acidentes com objetos perfurocortantes envolvem uma integração da biossegurança e das atividades de saúde dos trabalhadores (MARZIALE, 2007). A organização da saúde do trabalhador inclui uma coordenação e núcleos de serviços especializados em saúde do trabalhador. Um primeiro serviço dessa natureza foi criado em 1986. Tais instâncias são responsáveis pela programação de atividades de promoção de saúde dos trabalhadores, incluindo a notificação de acidentes de trabalho por meio de instrumento único definido pela própria instituição. 
Os objetivos do estudo foram: (i) analisar a qualidade de registros em acidentes biológicos causados por objetos perfurocortantes; (ii) analisar os subsequentes fluxos de tratamento, notificação, acompanhamento, profilaxia e encaminhamento dos trabalhadores de saúde provenientes da própria instituição e de outras unidades de saúde da área metropolitana do Rio de Janeiro; (iii) apresentar uma proposta de vigilância para acidentes com perfurocortantes.

\section{Metodologia}

Estudo descritivo e retrospectivo sobre a qualidade dos registros de acidentes com material perfurocortante, entre 1999 e 2004, realizado em dois hospitais (Instituto de Pesquisa Evandro Chagas - Ipec e Instituto Fernandes Figueira - IFF) e um laboratório (Laboratório de Referência Nacional de Hepatite Viral - LRN-HV) de uma mesma instituição de saúde. Ambos os hospitais incluíam em suas atividades o tratamento dos trabalhadores de saúde que se acidentavam com materiais perfurocortantes.

Para o estudo, foi elaborado um questionário semiestruturado, que foi respondido pelos 14 funcionários envolvidos nos registros dos acidentes nos três setores estudados. Os dados coletados a partir dos diferentes instrumentos de registro foram tabulados em um banco e analisados por meio do software SPSS (versão 16.0).

O presente estudo foi aprovado pelo Comitê de Ética em Pesquisa da Escola Nacional de Saúde Pública Sérgio Arouca (ENSP-Fiocruz), conforme as normas do Ministério da Saúde (BRASIL, 1997a), sob o número 42/05.

\section{Resultados e discussão}

No hospital materno-infatil IFF, até 2002, os registros com acidentes biológicos eram processados pela Comissão de Infecção Hospitalar (CIH) e pela farmácia, representadas pelo mesmo funcionário. Desde 2002, os registros têm sido processados também pelo Núcleo de Saúde do Trabalhador do IFF (Nustiff), constituído por cinco membros. Havia também um funcionário encarregado da biossegurança hospitalar que ministrava cursos afins na unidade. Os acidentes causados por materiais perfurocortantes eram registrados em formulários diversos, de acordo com os setores: Nustiff, CIH, Farmácia, Núcleo de Saúde do Trabalhador da Fundação Oswaldo Cruz (Nust) e no formulário da Secretaria Municipal de Saúde do Rio de Janeiro (SMS-RJ).
O Ipec, unidade voltada para a pesquisa clínica, o ensino e o tratamento de doenças infecciosas, possuía em seu organograma uma divisão de vigilância de saúde com um setor específico voltado para a saúde dos trabalhadores internos e da área metropolitana do Rio de Janeiro. O Ipec utilizava os seguintes formulários: SMS-RJ, Nust, Sinan e o Registro de Acidente de Trabalho da Coordenação de Saúde do Trabalhador (CST/Fiocruz).

No Laboratório de Referência Nacional de Hepatites Virais (LRN-HV), todos os funcionários e egressos recebiam treinamento prévio em biossegurança e registravam os acidentes detectados em formulários do Nust e da SMS-RJ.

Portanto, constatou-se que o formulário do Nust, instrumento oficial responsável pelo registro de acidentes, incluindo perfurocortantes, entre trabalhadores da instituição, estava presente nos três setores analisados. Contudo, cada setor também fazia uso de instrumentos próprios e da SMS-RJ. Portanto, os registros, possuíam informações distintas e às vezes incompletas e/ou inadequadas. Como consequência direta, foi constatado um grande volume de formulários e relatórios. Houve também a constatação de subnotificações, conformes relatos dos profissionais notificadores durante a aplicação do questionário semiestruturado, sendo confirmado quando se aferiu os dados das unidades estudadas com o setor responsável pela Saúde do Trabalhador (Nust), e especificamente quando comparados com os dados da SMS-RJ. Observou-se também inadequação no preenchimento dos formulários, ressaltando a insuficiência de dados acerca dos acidentados e dos respectivos acidentes, conforme já observado em outros estudos (BRAGA, 2000; BRANDÃO JUNIOR, 2000).

No IFF, os acidentes causados por materiais perfurocortantes eram registrados em cinco diferentes formulários: Nustiff, CTBio, Farmácia, Nust e SMS-RJ. O Ipec empregava quatro instrumentos de registros: Serviço de Orientação em Saúde do Trabalhador (SOS), Nust, SMS-RJ e Sinan. Finalmente, o LRN-HV registrava os acidentes em formulários do Nust e da SMS-RJ.

Comparação entre os formulários utilizados nos setores estudados mostrou que o formulário da SMS-RJ apresentava maior completude de informações do que o formulário do Nust, acrescido ainda de informações relevantes, como: o acompanhamento dos trabalhadores de saúde acidentados, o paciente-fonte, data, descrição e relatório do acidente. Quanto aos demais formulários utilizados, não foi verificada padronização de seus conteúdos: as informações eram variadas e incompletas para o tipo de acidente abordado neste trabalho (Tabela 1). 
Tabela 1 Conteúdo presente nos formulários de informações dos acidentes biológicos causados por material perfurocortante usados pelas unidades pesquisadas, Rio de Janeiro, RJ, 1999 a 2004

\begin{tabular}{|c|c|c|c|c|c|}
\hline Variáveis (presença de) & SOS & $S M S-R J$ & Nustiff & Nust & Sinan \\
\hline 1 - Data do acidente & $x$ & $x$ & $x$ & $x$ & $\mathrm{x}$ \\
\hline 2 - Hora & & $x$ & $\mathrm{X}$ & $x$ & $\mathrm{X}$ \\
\hline 3 - Local onde ocorreu o acidente & & $x$ & $x$ & $x$ & \\
\hline 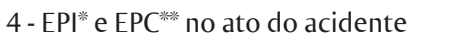 & & & $x$ & $x$ & \\
\hline \multicolumn{6}{|l|}{5 - Treinamentos para uso de EPI e EPC } \\
\hline \multicolumn{6}{|c|}{6 - Como é feito o descarte do material perfurocortante } \\
\hline 7 - Data da notificação & $\mathrm{X}$ & $x$ & $x$ & $x$ & $x$ \\
\hline 8 - Hora da notificação & $x$ & $x$ & & & \\
\hline 9 - Como aconteceu o AT橉 & $x$ & $x$ & $x$ & $x$ & $x$ \\
\hline 10 - Objeto causador & $x$ & & & & \\
\hline 11 - Fluido orgânico envolvido & $x$ & $x$ & & & \\
\hline 12 - Local de exposição & & & $\mathrm{X}$ & $x$ & $\mathrm{X}$ \\
\hline \multicolumn{6}{|l|}{13 - Procedimentos que realizava no AT } \\
\hline 14 - Encaminhamento dado & $x$ & $x$ & & & \\
\hline 15 - Conduta pós-acidente & & $\mathrm{X}$ & & & \\
\hline 16 - Exame laboratorial solicitado & & $\mathrm{X}$ & & & \\
\hline 17 - Perfil sorológico do trabalhador & & $\mathrm{X}$ & & & \\
\hline 18 - Perfil vacinal do trabalhador & & $\mathrm{X}$ & & & \\
\hline 19 - Exame solicitado & & $\mathrm{X}$ & & & \\
\hline \multicolumn{6}{|l|}{20 - Local onde foi realizado o exame } \\
\hline 21 - Situação sorológica do paciente & & $\mathrm{X}$ & & & \\
\hline \multicolumn{6}{|c|}{ "EPI - Equipamento de Proteção Individual; *** EPC - Equipamento de Proteção Coletiva; ${ }^{* * * * * *}$ AT - Acidente de Trabalho } \\
\hline \multicolumn{6}{|c|}{ SOS - Serviço de Orientação em Saúde do Trabalhador } \\
\hline \multicolumn{6}{|c|}{ SMS-RJ - Secretaria Municipal de Saúde do Rio de Janeiro } \\
\hline \multicolumn{6}{|c|}{ Nustiff - Núcleo de Saúde do Trabalhador do Instituto Fernandes Figueira } \\
\hline \multicolumn{6}{|c|}{ Nust - Núcleo de Saúde do Trabalhador da Fundação Oswaldo Cruz } \\
\hline Sinan - Sistema de Informação de Agravos & da & & & & \\
\hline
\end{tabular}

Esta múltipla variação de instrumentos de registro põe em risco a determinação precisa do número real de acidentes causados por materiais perfurocortantes, limitando as respectivas medidas de promoção da saúde dos trabalhadores (SILVA, 2006; RAPPARINE, 2007).

Entretanto, mesmo no formulário de notificação do Nust, pode-se observar a ausência de dados relevantes, como: treinamento no uso de equipamentos de proteção individual (EPI) e de proteção coletiva (EPC), questionamentos acerca dos procedimentos de expurgo de objetos perfurocortantes usados e indicação dos possíveis patógenos envolvidos.
Durante o período do estudo, foram notificados 134 registros de acidentes de trabalho causados por materiais perfurocortantes, sendo que 120 eram trabalhadores oriundos das três unidades estudadas e 14 eram trabalhadores externos que foram atendidos pelo SOS do Ipec. Os casos de subnotificação foram atribuídos, principalmente, à falta de organização na utilização dos formulários e dos fluxos de encaminhamento, realizada pelos notificadores, além do medo de perder o emprego ou o cargo, por parte das vítimas, ou ainda à pouca importância atribuída à saúde dos trabalhadores, conforme também identificado por Jagger e Perry (2001) e pelos Centers for Disease Control and Prevention (2001). 
Outros autores acharam resultados semelhantes. Lima (2001) confirmou a inexistência ou informações insuficientes de acidentes entre trabalhadores em dois hospitais de Belém (PA) devido a fatores culturais, assim como relatórios com atrasos de até 12 meses, que, segundo o autor, poderiam ter sido reduzidos através de campanhas de conscientização promovidas pela instituição.

Napoleão et al. (2000) descobriram uma taxa de informação insuficiente de 91,9\% para acidentes com materiais biológicos entre o pessoal de enfermagem em instituições de assistência de saúde, relacionando o problema a medo de perda de emprego.

No presente estudo, dos 14 indivíduos responsáveis pelo registro das informações acerca dos acidentes de trabalho com exposição a material biológico, 9 trabalhavam no IFF, 4 no Ipec e 1 no LNR-HV. O perfil observado demonstrou a seguinte composição: 12 eram do sexo feminino e 2 do masculino; 9 eram funcionários públicos permanentes, enquanto os outros 5 possuíam outros vínculos empregatícios; 10 estavam em semanas de trabalho de 40 horas, $2 \mathrm{em}$ 30 horas, e os outros 2 em 20 horas. Todos aqueles que cumpriam carga horária semanal inferior a 40 horas possuíam outros empregos.

Dos 14 notificadores, 11 tinham ensino universitário completo e 3 , ensino secundário completo. A experiência profissional, porém, variava, mostrando que a função de registrar acidentes causados por materiais perfurocortantes não requeria experiência profissional específica. De fato, a revisão da literatura (BRAGA, 2000; BRANDÃO JUNIOR, 2000) mostrou a ausência de necessidade de um grupo de profissional específico para registrar tais acidentes de trabalho.

Os CDC dos Estados Unidos relatam a experiência bem-sucedida de inclusão de voluntários para reportar esses tipos de acidentes. De acordo com os CDC, a notificação deve ser ato consciente e compulsório entre os trabalhadores e gestores das instituições, conquistada pelo amadurecimento do processo de educação no trabalho, não requerendo formação específica (CENTERS FOR DISEASE CONTROL AND PREVENTION, 1987).

Como forma de conscientização do processo de trabalho, a instituição deste estudo oferece, bianualmente, um curso em biossegurança para os seus trabalhadores, e só recentemente foi incorporado a esse curso um novo módulo sobre a importância da notificação dos acidentes biológicos. Nele, estão incluídos: o preenchimento correto do formulário do Nust, fluxos a serem seguidos e o local para onde os trabalhadores acidentados deverão ser encaminhados, segundo a CST/Fiocruz. Portanto, os autores acreditam e compactuam com os CDC (CENTERS FOR DISEASE
CONTROL AND PREVENTION, 1987), segundo os quais o processo educativo sobre a temática trará mais eficácia no processo da notificação na referida instituição.

A Norma Regulamentadora no 32 (NR 32) do Ministério do Trabalho e Emprego recomenda que o trabalhador autorize os trabalhos e promova educação permanentemente, de forma que suas atividades laborais sejam mais seguras (BRASIL, 2008).

As diretrizes para os registros, o fluxo de acompanhamento e o tratamento para acidentes causados por material perfurocortante são elaboradas pelo Nust, exceto para tratamento específico de HIV e das hepatites B e C, quando são seguidas aquelas preconizadas pelo Ministério da Saúde (BRASIL, 2000b).

Conforme observado acerca dos trabalhadores que se acidentavam provenientes dos setores estudados, quando se tratava de acidente com material perfurocortante, deveriam informar imediatamente à divisão médica respectiva de sua unidade ou se encaminhavam à unidade de referência, o Ipec, que recebia, também, trabalhadores de saúde da região metropolitana. Durante a avaliação médica, seria realizado o teste rápido para HIV e solicitados os exames laboratoriais para as hepatites B e C do acidentado e do indivíduo-fonte (quando este último era conhecido e havia autorizado a retirada de uma amostra de sangue), seguido da prescrição do tratamento com medicamentos antirretrovirais.

Como observado anteriormente, porém, o acidente não era sempre registrado de imediato - foram identificados dois casos de registros datados de um ano após o acidente, verificado pela data contida no formulário de uma das unidades hospitalares. As unidades também mostraram diferenças relativas ao protocolo na administração da medicação para os acidentados e à duração do tratamento antirretroviral.

Em seguida, o trabalhador acidentado seguiria para a farmácia do Ipec e do IFF para receber o medicamento e começar o tratamento até duas horas depois da exposição, de acordo com as diretrizes do Ministério da Saúde (BRASIL, 1996) e as recomendações pelo Programa STD/AIDS da Saúde Municipal do Rio de Janeiro (SÃO PAULO, 2002). Ambas as farmácias, porém, não mantinham registros específicos referenres à distribuição dos medicamentos antirretrovirais para acidentes causados por material perfurocortante.

O próximo passo seria informar o acidente ao Nust. Contudo, esse procedimento não ocorreu de forma satisfatória, conforme observado durante as comparações entre os bancos de dados do Nust e das unidades de estudo, assim como não observamos correlação com os relatórios de acidente ou acompanhamento. 
Tomando por base os trabalhadores que haviam recebido treinamento em biossegurança e sofrido acidentes causados por materiais perfurocortantes, constatamos orientação apenas para o uso de EPI e manipulação de fluidos corporais.

Infelizmente, não havia registros se os trabalhadores acidentados, assim como os pacientes-fonte, de fato, se encaminhavam ao laboratório para submissão dos exames prescritos. Em uma das unidades, o farmacêutico responsável pela dispensação dos medicamentos antirretrovirais relatou que alguns trabalhadores acidentados de forma percutânea às vezes não retornavam, permanecendo sem o tratamento específico para HIV, comprometendo a vigilância dos trabalhadores de saúde com HIV/ AIDS como descrito pelo CDC (CENTERS FOR DISEASE CONTROL AND PREVENTION, 2011).

Como esse fluxo ainda não está incorporado na rotina de todos os setores da instituição, exceto no Ipec, acreditamos que este procedimento poderia estar presente nos formulários de registro para melhorar o monitoramento da saúde dos acidentados pelo Nust. Para corrigir essa falha, todos os setores de assistência médica da instituição deveriam preparar e seguir os procedimentos operacionais padrão (POP) para pacientes acidentados, conforme recomendação da Occupational Safety and Health Administration (2001), dos Estados Unidos.
A profilaxia pós-exposição de trabalhadores de saúde que sofreram acidentes causados por material perfurocortante (Figura 1) mostrou que $64(47,76 \%)$ não receberam qualquer profilaxia, $32(23,88 \%)$ receberam profilaxia de HIV e 12 (8,96\%) receberam profilaxia de HIV e HBV, demonstrando a importância de testar os pacientes-fontes e a necessidade de imunização dos trabalhadores de saúde.

Essa múltipla forma de registro desconectada põe em risco a determinação precisa do número real de acidentes causados por material perfurocortante, assim como os resultados, limitando as respectivas medidas de promoção de saúde dos trabalhadores (SILVA, 2006a; RAPPARINE, 1998; UNIVERSIDADE DE SÃO PAULO, 2003).

Por ocasião da comunicação do acidente ao Nust, essa etapa ocorreu de forma insatisfatória nos três setores. Quando comparado o registro do banco de dados do Nust com os setores analisados, verificou-se que, dos 134 acidentes notificados no período estudado, foram confirmados apenas 21 casos $(15,67 \%)$, corroborando as subnotificações quanto aos registros de acidentes.

A necessidade de uma proposta de vigilância de saúde dos trabalhadores passou a ser uma consequência no processo educativo e construtivo para o monitoramento dos acidentes biológicos da instituição.

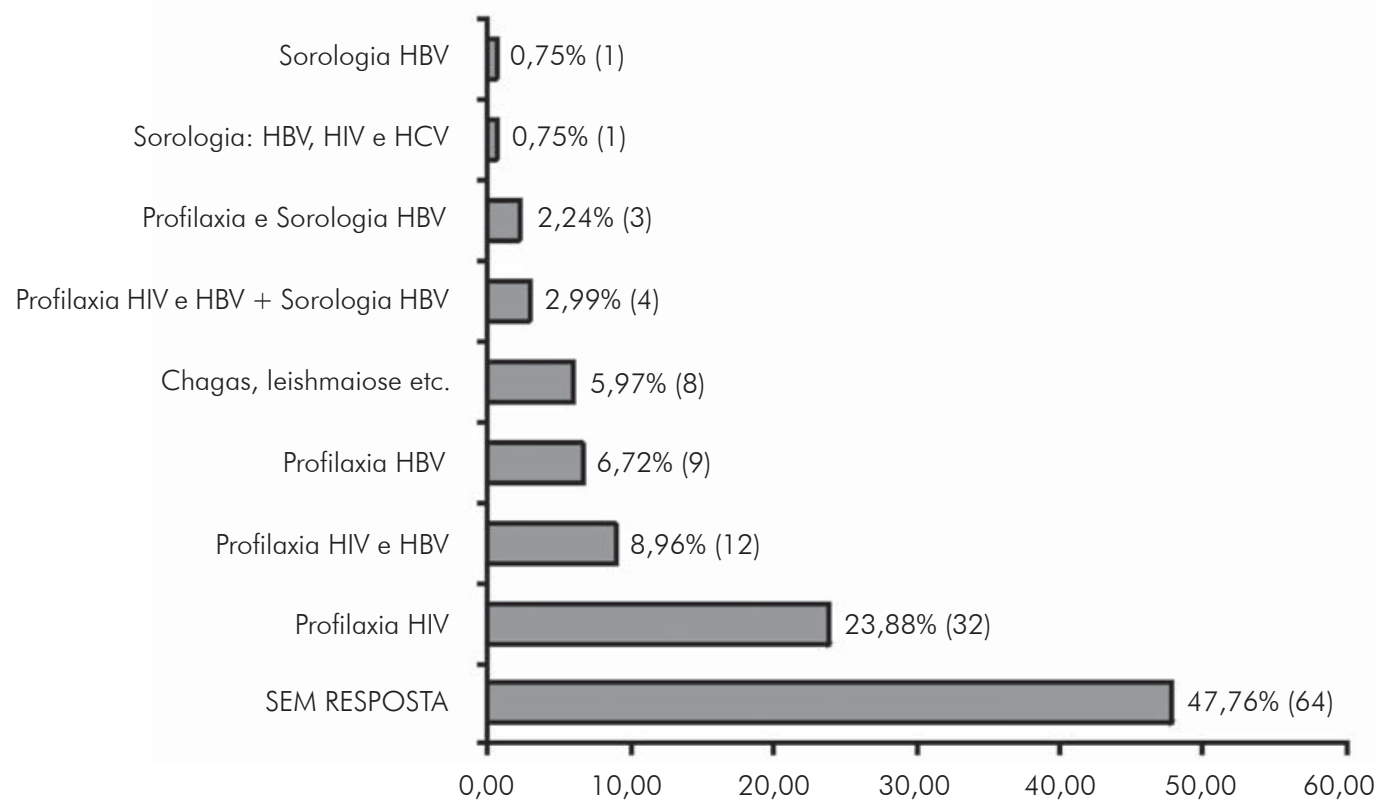

Figura 1 Condutas pós-acidentes biológicos causados por material perfurocortante registrados nas Fichas de Notificação pelas unidades pesquisadas, no Rio de Janeiro, RJ, no período de 1999 a 2004 
Proposta para vigilância de saúde dos trabalhadores da instituição estudada

- Uso de formulário de informações e pesquisa para acidentes causados por material perfurocortantes reestruturado, baseado nas variáveis presentes nas fichas do Sinan e SMS-RJ e que não estão contempladas no formulário do Nust (Tabela 1). Para monitoramento de qualidade pelo CST, os novos formulários deverão ser incorporados à Rede de Informações em Saúde, Trabalho e Ambiente (Rista) (SILVA, 2006), permitindo uma dinâmica mais modernizada e de melhor interação entre as várias unidades da instituição e a CST;

- Implantação de medidas de vigilância em saúde do trabalhador para acidentes causados por materiais perfurocortantes, conforme referido em estudo sobre a saúde do trabalhador da saúde (OSÓRIO, 2005);

- Adoção de medidas de pré-exposição, conectadas a atividades educacionais (MARZIALE, 2010). Portanto, o uso obrigatório de EPI e EPC, incluindo procedimentos para descartar materiais potencialmente infectados, requer treinamento periódico, direcionado para melhorar a sua utilização e ajudar a reduzir a exposição dos trabalhadores de saúde a fluidos sanguíneos e/ou corporais enquanto os manuseiam. Braga (2000) e Brandão Junior (2000) já identificaram a necessidade de tais iniciativas, tendo demonstrado a falta de treinamento específico na administração segura de pacientes nas unidades hospitalares, e recomendaram que os trabalhadores nas instituições de saúde tivessem um cartão de vacinação e acompanhamento periódico de marcadores sorológicos para patógenos específicos que pertençam às respectivas atividades de trabalho;

- Adoção de medidas de pós-exposição, quanto aos medicamentos recomendadas pelo Ministério da Saúde do Brasil (NAPOLEÃO et al., 2000) para uso em profilaxia de pós-exposição, devem estar disponíveis, reforçadas por atividades coletivas e educacionais em um formato contínuo;

- Implantação de uma Comunidade Virtual no Ambiente Interno da instituição. Esta proposta envolve o desenvolvimento de um foro de debate aberto, voluntário, criativo e dinâmico sobre o tema de acidentes causados por materiais perfurocortantes, que permitirá uma discussão interna acerca dos riscos biológicos entre trabalhadores de saúde. O debate pode ser sustentado através de listas de chat e de e-mail sobre temas e assuntos ligados direta ou indiretamente à saúde dos trabalhadores e ao controle de infecção. O referido debate teria a participação dos trabalhadores com diferentes experiências profissionais e de diferentes unidades, contribuindo para estabelecer um sistema de vigilância para acidentes com materiais biológicos.

\section{Conclusões}

Em síntese, a análise dos formulários de informações e o perfil de indivíduos que registraram os acidentes causados por materiais perfurocortantes nos três setores estudados mostrou:

- A ausência de formação dos notificadores para desempenhar a função e uso de diferentes formulários de registro e investigação, administração e acompanhamento dos acidentes causados por material perfurocortante; discordância e subnotificação, conforme demonstrado por comparação entre bancos de dados e os formulários utilizados, o do Nust e o da SMS-RJ.

- A necessidade de implantar medidas de conscientização para gerentes e profissionais envolvidos em informação em saúde, com ênfase nas medidas preventivas em relação às hepatites virais $\mathrm{B}$ e $\mathrm{C}$ e a AIDS por meio de cursos educacionais permanentes, na modalidade a distância (BRASIL, 1996).

\section{Contribuições de autoria}

Silva, A. I. D.: elaborou e delineou o projeto e foi a responsável pelo levantamento de dados, pela análise e interpretação dos resultados e pela elaboração do manuscrito. Machado, J. M. H.: participou da elaboração, do delineamento, da orientação e da análise do projeto, da revisão crítica e da aprovação da versão final do texto. Santos, E. G. O. B.: contribuiu com a orientação da execução do projeto, a análise e a elaboração do manuscrito. Marziale, M. H. P.: participou na elaboração e na revisão da versão final publicada. 


\section{Referências}

BRAGA, D. Acidente de trabalho com materiais biológico em trabalhadores da equipe de enfermagem do Centro de Pesquisas Hospital Evandro Chagas: um olhar da saúde do trabalhador. 2000. 86 f. Dissertação (Mestrado em Saúde Pública)-Escola Nacional de Saúde Publica Fundação Oswaldo Cruz, Rio de Janeiro, 2000.

BRANDÃO JUNIOR, P. S. Biossegurança e AIDS: as dimensões psicossociais do acidente com material biológico no trabalho em Hospital. 2000. $124 \mathrm{f}$. Dissertação (Mestrado em Saúde Pública)-Escola Nacional de Saúde Publica Fundação Oswaldo Cruz, Rio de Janeiro, 2000.

BRASIL. Decreto no 2.172, de 5 de março 1997. Aprova o Regulamento dos Benefícios da Previdência Social. Diário Oficial [da] República Federativa do Brasil, Poder Executivo, Brasília, DF, 06 mar. 1997. Seção I, p. 4279. Disponível em: < http://www010.dataprev.gov.br/sislex/ paginas/23/1997/2172.htm>. Acesso em: 03 dez. 2011

. Lei n⿳⺈ 8.213/91, de 24 de jul. 1991. Dispõe sobre os Planos de Benefícios da Previdência Social e dá outras providências. Diário Oficial [da] República Federativa do Brasil, Brasília, DF, de 25 jul. 1991 e Republicado em 14 ago. 1998.

. Ministério da Saúde. Procedimentos frente a acidentes de trabalho com exposição a materiais potencialmente contaminados com o vírus da AIDS. Boletim Epidemiológico AIDS, v. 10, p. 3-5, 1996.

Ministério da Saúde. Normas éticas em pesquisas envolvendo seres humanos. Diário Oficial [da] República Federativa do Brasil, Poder Executivo, Brasília, DF, 23 set. 1997. Seção 1, p. 21118.

. Ministério da Saúde. Portaria Funasa

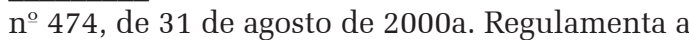
coleta de dados, fluxo e periodicidade de envio das informações sobre óbitos para o Sistema de Informações sobre Mortalidade - SIM. Disponível em: <http://www.funasa.gov.br/internet/arquivos/ legislacao/portariasFunasa/portaria_474_2000.PDF $>$. Acesso em: 03 dez. 2011.

. Ministério da Saúde. Manual de condutas de exposição ocupacional a materiais biológicos: Hepatite e HIV. Brasília: Ministério da Saúde, 2000b.

. Ministério da Saúde. Secretaria de Vigilância em Saúde. Sistema de Informação de Agravos de Notificação - Sinan. 2.ed. Brasília: Ministério da Saúde, 2007. (Série A. Normas e Manuais Técnicos).

Ministério do Trabalho. Risco biológico: guia técnico. Os riscos biológicos no âmbito da Norma Regulamentadora $n^{\circ} 32$. Brasília: Ministério do Trabalho, 2008. Disponível em: <http://www.mte.gov. br/seg_sau/guia_tecnico_cs3.pdf $>$. Acesso em: 05 maio 2011.
CENTERS FOR DISEASE CONTROL AND PREVENTION. Recommendations for prevention of HIV transmission in health care settings. Morbity and Mortality Weekly Report (MMWR), v. 36, 1987.

. Updated U.S. Public Health Service Guidelines for the Management of Occupational Exposures to HBV, HCV, and HIV and Recommendations for PostExposure Prophylaxis. Morbity and Mortality Weekly Report (MMWR), v. 50, 2001.

. Worker health chartbook, 2004. Cincinnati: National Institute for Occupational Safety and Health, 2004. DHHS (NIOSH) Publication No. 2004-146. Disponível em: <http://www.cdc.gov/niosh/docs/2004146/ch2/ch2-2.asp.htm>. Acesso em: 05 maio 2011.

. Division of healthcare quality promotion. Surveillance of healthcare personnel with HIV/AIDS [serial online]. Disponível em: <http://www.cdc.gov/ ncidod/hip/Blood/hivpersonnel.htm > . Acesso em: 21 set. 2011.

COELHO, H. Biossegurança hospitalar. In: MARTINS, E. V; SILVA, F. A. L; LOPES, M. C. M. (Ed.).

Biossegurança, informações e conceitos: textos básicos. Rio de Janeiro: Fiocruz; 2006. p. 288.

GODFRE, K. Sharp practice. Nursing Times, v. 97, p. 22-24, 2001.

JAGGER, J. B.; PERRY, J. Risk phlebotomy with a syringe. Nursing, v. 31, n. 2, p. 73, 2001.

LIMA, V. L. de A. Em casa de ferreiro o espeto é de pau: uma proposta para o sistema de vigilância nos Hospitais da Fundação Santa Casa de Misericórdia do Pará e Hospital Universitário João de Barros Barreto. 2001. 89 f. Dissertação (Mestrado em Saúde Pública) -Escola Nacional de Saúde Publica Fundação Oswaldo Cruz, Rio de Janeiro, 2001.

MARZIALE, M. H. P. et al. Subnotificação de acidentes de trabalho com material perfurocortante entre trabalhadores de enfermagem brasileiros. Revista Brasileira de Enfermagem, v. 56, n. 2, p. 164-168, mar./ abr. 2003.

. et al. Acidente com material biológico em hospital da rede de prevenção de acidentes do trabalho - REPAT. Revista Brasileira de Saúde Ocupacional, São Paulo, v. 32, n.115, p. 109-110, jan./fev. 2007.

. et al. Rede de prevenção de acidentes de trabalho: uma estratégia de ensino a distância. Revista Brasileira de Enfermagem, v. 63, n. 2, p. 250-256, mar./ abr. 2010.

MARZIALE, M. H. P.; RODRIGUES, C. M. A produção científica sobre os acidentes de trabalho com material perfurocortante entre trabalhadores de enfermagem. Revista Latino-Americana de Enfermagem, v. 10, p. 571-577, 2002. 
MINAYO, M. C. de S. Proposta para uma política institucional. Rio de Janeiro: Fundação Oswaldo Cruz, 1997.

. et al. Fiocruz saudável: uma experiência institucional. Ciência e Saúde Coletiva, v. 3, n. 2, p. 151-161, 1998.

NAPOLEÃO, A. A. et al. Causas de subnotificação de acidentes do trabalho entre trabalhadores de enfermagem. Revista Latino-Americana de Enfermagem, v. 8, p. 119-20, 2000.

OCCUPATIONAL SAFETY AND HEALTH

ADMINISTRATION. 29 CFR Part 1910.1030.

Occupational Exposure to Blood borne Pathogens; Needlesticks and Other Sharps Injuries; Final rule. Fed Register, v. 66, p. 5317-5325, 2001.

OSORIO, C. et al. Proposição de um método de análise de análise coletiva dos acidentes de trabalho no hospital. Cadernos de Saúde Pública, v. 21, n. 2, p. 517-524, 2005.

RAPPARINE, C. Acidentes por materiais biológicos. Revista em Foco, v. 7, p. 45-49, 1998.

. et al. Occupationally acquired infectious diseases among health care workers in Brazil: use of Internet tools to improve management, prevention and surveillance. American Journal of Infection Control, v. 35, p. 267-70, 2007.

SÃO PAULO (Estado). Secretaria da Saúde. Programa DST/AIDS-SP. Vigilância de acidente com material biológico. Boletim Epidemiológico-SINABIO, v. 1, p. 20, 2002.

SILVA, A. I. C. D. da. Análise da qualidade dos registros de acidentes biológicos com materiais pérfurocortantes na Fundação Oswaldo Cruz no período de 1999 a 2004. 2006. 86 f. Dissertação (Mestrado em Saúde Pública)-Escola Nacional de Saúde Pública da Fundação Oswaldo Cruz, Rio de Janeiro, 2006.

. Desafios e perspectivas na implantação de uma ficha de notificação e investigação de acidentes biológicos para os trabalhadores de uma instituição pública de saúde da cidade do Rio de Janeiro. In: CONGRESSO BRASILEIRO DE INFORMAÇÃO EM SAÚDE, 10., 2006, Florianópolis: CBIS, 2006a. Disponível em: < http://www.sbis.org.br/cbis/ arquivos/819.pdf>. Acesso em: 05 maio 2011.

SPAGNUOLO, R. S.; BALDO, R. C. S.; GUERRINI, I. A. Análise epidemiológica dos acidentes com material biológico registrado no Centro de Referência em Saúde do Trabalhador - Londrina-PR. Revista Brasileira de Epidemiologia, v. 11, n. 2, p. 315-23, 2008.

UNIVERSIDADE DE SÃO PAULO. Escola de Enfermagem. Rede de prevenção de acidentes de trabalho com material biológico em hospitais brasileiros - REPAT. Ribeirão Preto: Universidade de São Paulo, 2003. Disponível em: < http://www.repat. eerp.usp.br>. Acesso em: 05 maio 2011. 